\title{
The Feasibility and Development of an In situ Ion Irradiation TEM at Sandia National Laboratories
}

\author{
K. Hattar*, P. Rossi*,†, G. Vizkelethy*, B.L. Doyle* \\ * Dept. of Radiation Solid Interactions, Sandia National Laboratories, Albuquerque, NM 87313 \\ ${ }^{\dagger}$ Dept. of Physics of the University and INFN, Padua, Italy
}

Radiation damage plays a significant role in limiting the lifetime of systems ranging from circuit elements in satellites to nuclear reactors. In order to understand the underlying physics governing these and other systems exposed to radiation environments, experimental observations of the microstructure during ion irradiation is needed. The field of in situ TEM ion irradiation is celebrating its $50^{\text {th }}$ anniversary with a renewed growth that includes the development of a facility at Sandia National Laboratories. During these five decades, the field has incorporated the latest technologies from both the ion accelerator and TEM communities. [1] The 11 current facilities worldwide (one in the US) with varying capabilities will be discussed and compared to the facility being developed at Sandia.

A three generation process is underway for the development of a unique in situ TEM ion irradiation facility at Sandia. The first generation of development seen in Figure 1 will entail the insertion of MeV-energy heavy ion beams produced by a Tandem accelerator into a JEOL 2100 $\mathrm{LaB}_{6}$ TEM perpendicular to the electron beam. The second generation will result in the concurrent flow of keV-energy light ions from a Colutron accelerator to the heavy ions introduced from the Tandem accelerator. In order to achieve this, a JEOL 2100 TEM was raised and aligned with a beam line port. In addition, the beam optics calculations were performed to optimize beam current and provide adequate dosimetery. To determine the feasibility of studying fields interested in events ranging from single ion strikes to greater than 300 dpa of damage, a simulation scheme was developed that incorporates ion species, energies, and geometry that will be utilized in the new in situ TEM ion irradiation facility. In order to predict the TEM micrograph produced from ion irradiation under the relevant conditions, a MARLOWE simulation was performed to predict that atom location after the ion irradiation and then formatted for JEMS image simulation. [2-3] An example of the JEMS high resolution image simulation of a GaAs film nearly seven nm-thick both before, Figure 2A, and after 1,000 ion cascades resulting from $30 \mathrm{MeV} \mathrm{Cu}$ implantations, Figure 2B.

This work was partially supported by the Division of Materials Science and Engineering, Office of Basic Energy Sciences, U.S. Department of Energy. Sandia National Laboratories is a multiprogram laboratory operated by Sandia Corporation, a wholly owned subsidiary of Lockheed Martin company, for the U.S. Department of Energy's National Nuclear Security Administration under contract DE-AC04-94AL85000.

References

[1] J.A. Hinks, NIMB 267 (209) 3652.

[2] MARLOWE review L. N. Brewer et al., Adv. Mater. 22 (2010) 1161.

[3] M.T. Robinson et al., Phys. Rev. B 9 (1974) 5008 


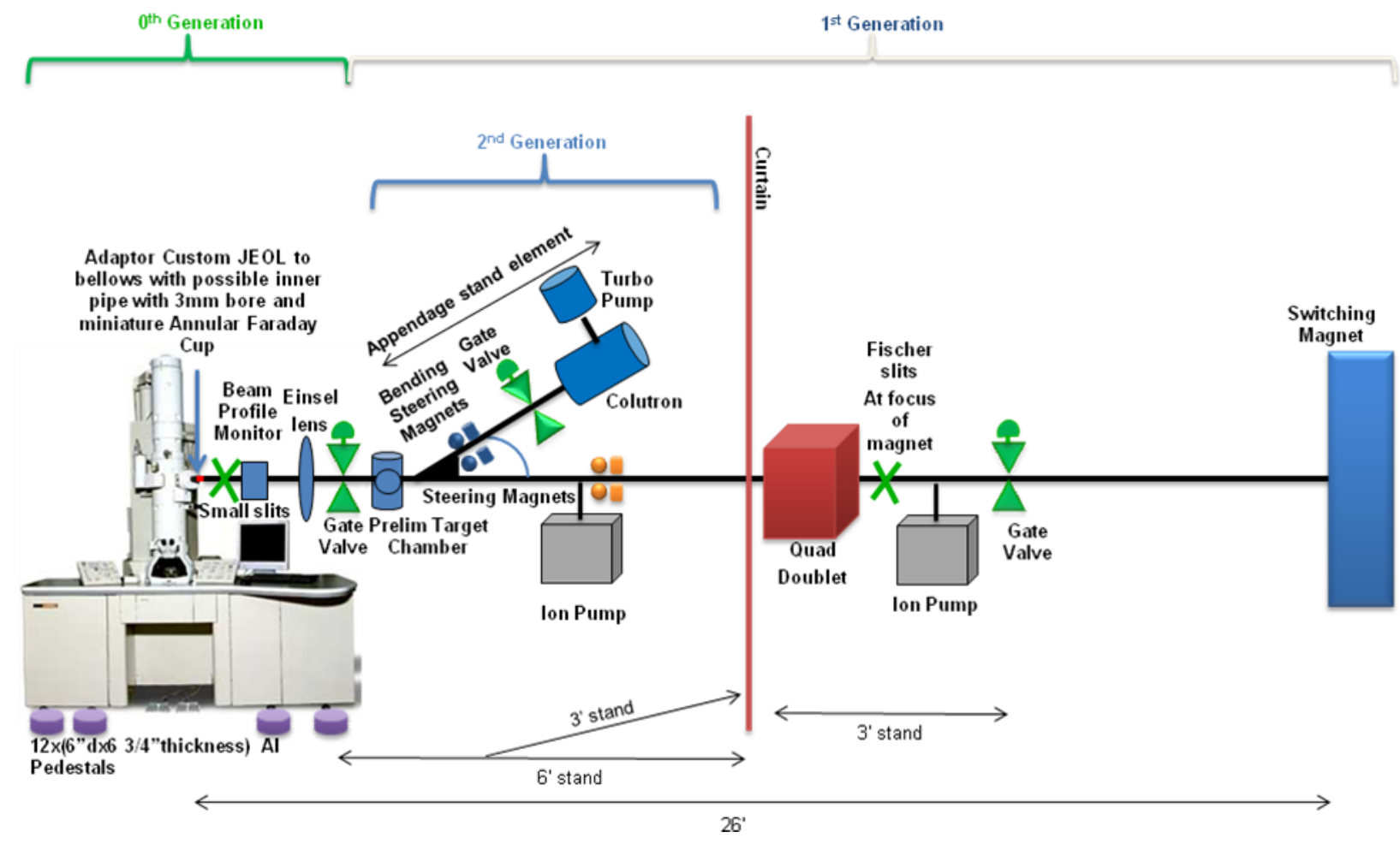

FIG. 1. A schematic of the ion beam line segments under construction that will permit in situ ion irradiation experiments with concurrent beams of light and heavy ions.

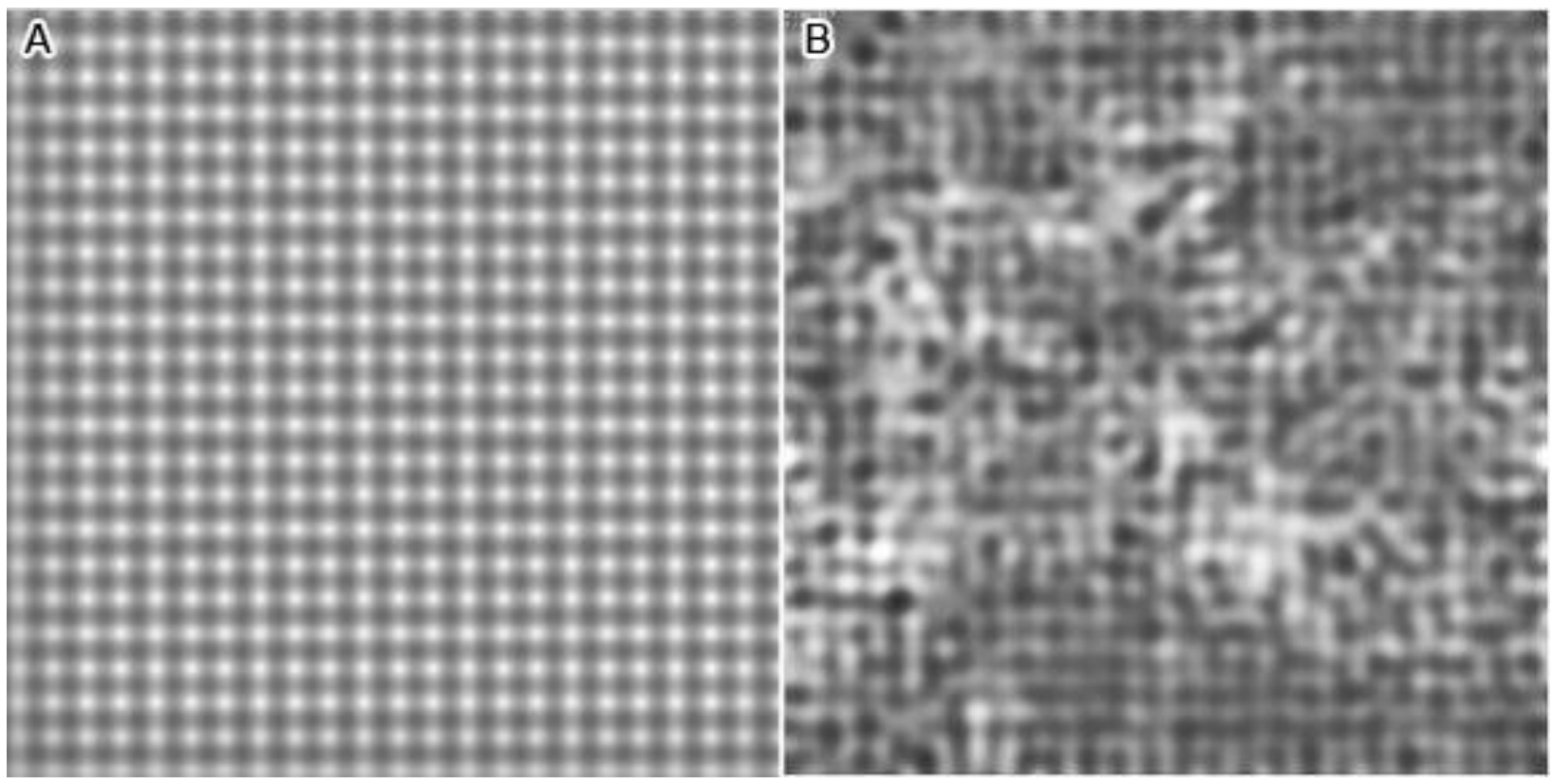

FIG. 2. A) JEMS high resolution TEM micrograph simulation of a perfect GaAs single crystal. B) JEMS TEM micrograph simulation of a GaAs structure predicted by a MARLOWE simulation after 1,000 cascades resulting from $30 \mathrm{MeV} \mathrm{Cu}$ implantations. 